\title{
The TT-TG Distance 40 Year-0ld Anniversary
}

\author{
Sebastien Zilber* \\ Department of Orthopedic and Traumatological Surgery, France \\ *Corresponding author: Sebastien Zilber, Department of Orthopedic and Traumatological Surgery, France
}

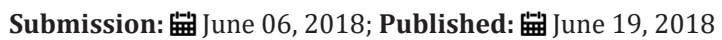

\begin{abstract}
Tibial tuberosity medialization could be proposed as a treatment for chronic patellar instability associated with an increased tibial tuberositytrochlear groove distance (TT-TG). TT-TG is 40 year old as it was first described by Goutallier et al. [1]. According to his further clinical researches, TT-TG should not be considered by itself when planning a tibial tuberosity medialization but correlate to the trochlear groove angle. This correlation allowed an optimization of the tibial tuberosity medialization technique by limiting the risk of persistent instability (TT not enough medialized) or pain (TT too medialized).
\end{abstract}

\section{Opinion}

The TT-TG was first described by Goutallier et al. [1] in Revue de chirurgie orthopédique. The measure was presented as being a quantification of the quadricipital unit valgus during the patella engagement in the trochlea. The high demanding technique as well as TT-TG measure results of 3 populations were exposed. The TTTG should be performed on a lying patient with a $30^{\circ}$ knee flexion without any rotation in the hip. Each knee should be radiographed separately as the central X-ray beam should be in the axe of the quadriceps insertion.

According to this technique, 70 non operated knees were included in a study. Sixteen of them were patients over the age of 65 without any knee dysfunction (control group), 30 knees had lateral femoro-patellar arthritis, 24 knees had patellar chronic instability. The tibial tuberosity was always lateral to the trochlear groove (positive TT-TG). The mean TT-TG distance was $13 \mathrm{~mm}$ for the control group (7-17mm), and $22 \mathrm{~mm}$ for the arthritis group (16$31 \mathrm{~mm})$ and the instability group (9-38mm).

The authors conclude that TT-TG distance appreciate one of the factors of the patella lateralization while engaging the trochlea and should have a therapeutic incidence. Since this time, the TTTG distance measurement was improved by using CT scan and MRI [2,3]. Izadpanah et al. [4] recently confirmed the necessity of a perfect joint positioning and a $30^{\circ}$ knee flexion during TTTG evaluation. Fifteen years later, Goutallier et al. [5] published the clinical results of 63 patients treated by tibial tuberosity medialisation associated with lateral patellar ligament section for chronic patellar instability in a French rheumatology journal which does not exist anymore. These results were also reported in a book dedicated to the femoro-patellar disorders in 1999 [6]. The postoperative clinical results of 63 patients with true chronic patellar instability (at least one femoro-patellar dislocation) were analyzed according to different mechanical factors: the post-operative TT-TG distance, the trochlear angle at $30^{\circ}$ knee flexion, the patella height, and the femoro-tibial axis. The mean follow-up since the procedure was 8 years [7-15]. Pain, subjective and objective instabilities were studied. There was a strong correlation between pain and postoperative TT-TG distance less than $3 \mathrm{~mm}$. there was no isolated mechanical factors correlated to the subjective and objective instability. However, the stability was strongly correlated to the post-operative TT-TG distance according to the trochlear angle.

Figure 1

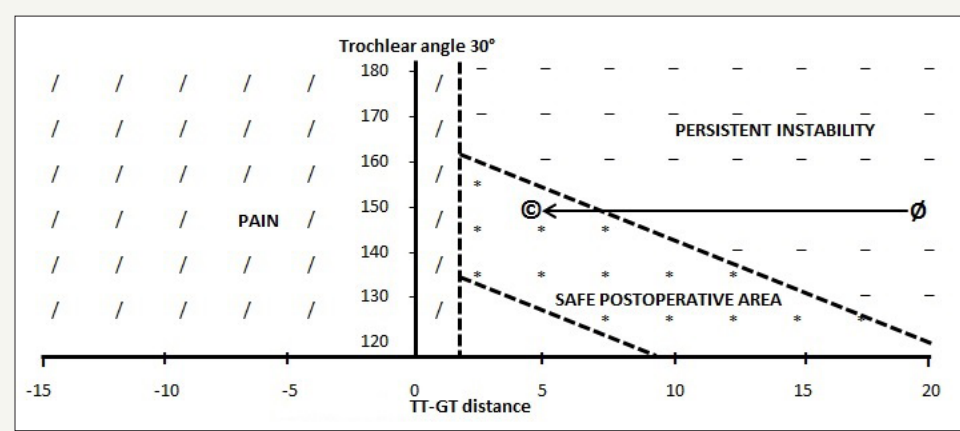


Therefore, a diagram showing the area were operated knees should be to avoid as much as possible persistent instability and postoperative pain was designed (Figure 1). The TT-TG medialization of the tibial tubercule should be quantified and is adapted to each patient. For example, a patient with chronic patellar instability and a TT-TG distance of $20 \mathrm{~mm}$ with a trochlear angle of $150^{\circ}$ should be medialized up to $15 \mathrm{~mm}$ to fit the "good results" area (Figure 1).

TT-TG distance is usually considered as pathologic if superior to $15-20 \mathrm{~mm}$ when associated to chronic patellar instability $[8,12]$. However, the consideration of the TT-TG as an absolute value was already questioned. Balcarek et al. [7] described a relative value of the TT-TG distance that is defined as the ratio between the TTTG distance and the width of the distal femur. Hingelbaum et al. [11] claimed that TT-TG distance should be correlated to the size of the knee introducing the TT-TG index that allows an evaluation of the necessity for TT-TG medialization on a more individualized basi. Ferlic et al. [9] found significant correlations between TT-TG distance and several parameters of knee joint size and leg length in patient with chronic patellar instability. The authors claim for application of indices determining TT-TG as a ratio of joint size instead of using TT-TG absolute values. Graf et al. [10] emphasized the need for considering TT-TG distance with tibial sulcus angle (TSA) to avoid overcorrection with TT-TG medialization. As Tsujimoto et al. [15] they found a significant positive correlation between the TT-TG distance and the Q-angle $[7,15]$. However, none of these studies were comparing postoperative findings according to the success or not of a TT-TG medialization.

Chronic patellar instability is most of the time the consequence of several anatomical pathologies that are entangled [13]. Each of these variations should be considered while planning a corrective surgery [14]. TT-TG distance was first described 40 years ago [1]. The unknown Goutallier's study of the correlation between postoperative TT-TG distance and TT-TG medialization clinical results according to the trochlear angle allows an optimization of this corrective surgery $[5,6]$. Thus, the medialization distance can be preoperatively quantified on an individualized basis to optimize the surgical result. The knowledge of this study could influence further works on chronic patellar instability and TT-TG medialization.

\section{References}

1. Goutallier D, Bernageau J, Lecudonnec B (1978) The measurement of the tibial tuberosity. Patella groove distanced technique and results (author's transl). Rev Chir Orthop Reparatrice Appar Mot 64(5): 423428.
2. Muneta T, Yamamoto H, Ishibashi T, Asahina S, Furuya K (1994) Computerized tomographic analysis of tibial tubercle position in the painful female patellofemoral joint. Am J Sports Med 22(1): 67-71.

3. Schoettle PB, Zanetti M, Seifert B, Pfirrmann CWA, Fucentese SF, et al. (2006) The tibial tuberosity-trochlear groove distance; a comparative study between CT and MRI scanning. The Knee 13(1): 26-31.

4. Izadpanah K, Weitzel E, Vicari M, Hennig J, Weigel M, et al. (2014) Influence of knee flexion angle and weight bearing on the Tibial Tuberosity-Trochlear Groove (TTTG) distance for evaluation of patellofemoral alignment. Knee Surg Sports Traumatol Arthrosc 22(11): $2655-2661$.

5. Goutallier D, Blachier D, Norotte G, Bernageau J (1993) Study of the different factors influencing the results of the treatment of patellar instability with luxation (s) treated by transposition of the tibial tuberosity and section of the external patellar flap. Rheumatology 45: 101-103.

6. Goutallier D, Beaufils P (1999) Pathologie fémoro-patellaire. Expansion Scientifique Publications, Paris, France.

7. Balcarek P, Jung K, Frosch KH, Stürmer KM (2011) Value of the tibial tuberosity-trochlear groove distance in patellar instability in the young athlete. Am J Sports Med 39(8): 1756-1761.

8. Dejour H, Walch G, Josserand NL, Guier C (1994) Factors of patellar instability: an anatomic radiographic study. Knee Surg Sports Traumatol Arthrosc 2(1): 19-26.

9. Ferlic PW, Runer A, Dirisamer F, Balcarek P, Giesinger J, et al. (2017) The use of tibial tuberosity-trochlear groove indices based on joint size in lower limb evaluation. Int Orthop 42(5): 995-1000.

10. Graf KH, Tompkins MA, Agel J, Arendt EA (2018) Q-vector measurements: physical examination versus magnetic resonance imaging measurements and their relationship with tibial tubercle-trochlear groove distance. Knee Surg Sports Traumatol Arthrosc 26(3): 697-704.

11. Hingelbaum S, Best R, Huth J, Wagner D, Bauer G, et al. (2014) The TT-TG Index: a new knee size adjusted measure method to determine the TTTG distance. Knee Surg Sports Traumatol Arthrosc 22(10): 2388-2395.

12. Koëter S, Diks MJF, Anderson PG, Wymenga AB (2007) A modified tibi12al tubercle osteotomy for patellar maltracking: results at two years. J Bone Joint Surg Br 89: 180-185.

13. Tecklenburg K, Dejour D, Hoser C, Fink C (2006) Bony and cartilaginous anatomy of the patellofemoral joint. Knee Surg Sports Traumatol Arthrosc 14(3): 235-240.

14. Thaunat M, Bessiere C, Pujol N, Boisrenoult P, Beaufils P (2011) Recession wedge trochleoplasty as an additional procedure in the surgical treatment of patellar instability with major trochlear dysplasia: early results. Orthop Traumatol Surg Res 97(8): 833-845.

15. Tsujimoto K, Kurosaka M, Yoshiya S, Mizuno K (2000) Radiographic and computed tomographic analysis of the position of the tibial tubercle in recurrent dislocation and subluxation of the patella. Am J Knee Surg 13(2): 83-88.
Creative Commons Attribution 4.0 International License

For possible submissions Click Here

\section{Submit Article}

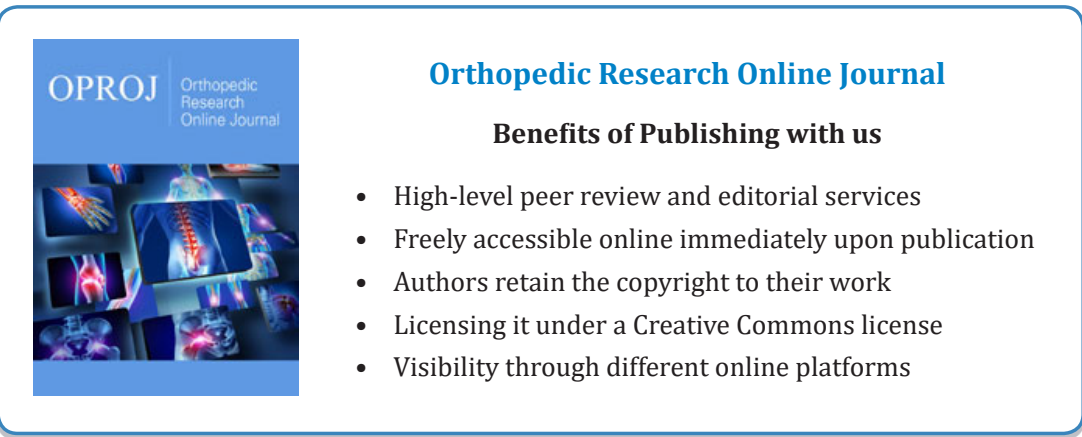

